\title{
European and American Guidelines for Multiple Sclerosis Treatment
}

\author{
Angelo Ghezzi
}

Received: July 17, 2018 / Published online: October 12, 2018

(C) The Author(s) 2018

\section{ABSTRACT}

The management and treatment of multiple sclerosis (MS) is becoming more and more complex as many medications are now available, with different routes of administration, mechanisms of action, and effectiveness and safety profiles. The decision-making process to choose the right medication is a complex task requiring a careful evaluation of the results of clinical and post-marketing studies, and the capability to translate data from clinical studies to patients in everyday clinical practice. Two European neurological societies, the European Committee for Treatment and Research in Multiple Sclerosis (ECTRIMS) and European Academy of Neurology (EAN), and the American Academy of Neurology (AAN) have recently delivered guidelines for MS treatment, helping neurologists to address the most common clinical issues related to this topic. The two guidelines offer a similar view and similar recommendations for the most relevant and common questions of clinical practice. For some aspects of MS treatment the statements

Enhanced digital features To view enhanced digital features for this article go to https://doi.org/10.6084/ m9.figshare.7098752.

\section{A. Ghezzi $(\square)$}

Centro Studi Sclerosi Multipla, Ospedale di

Gallarate, Gallarate, Italy

e-mail: angelo.ghezzi@asst-valleolona.it are slightly different, in particular regarding pregnancy management in relation to treatments, the use of mitoxantrone, and the treatment of secondary progressive MS (SP-MS).

Keywords: Guidelines; Multiple sclerosis; Therapy; Recommendations

\section{COMMENTARY}

The management and treatment of multiple sclerosis (MS) is becoming more and more complex: after the approval of interferon beta$1 \mathrm{~b}$ (IFNB) as the first disease-modifying drug (DMD) about 2 decades ago, many medications are now available for the treatment of MS, with different routes of administration (oral, i.m., s.c., i.v.), different mechanisms of action, and different effectiveness and safety profiles [1].

This impressive development of knowledge has dramatically changed the management of MS:

- Physicians now have a wide spectrum of medications at their disposal, with the possibility, in discussion with the patient, to select the right medication (in relation to mechanism, route of administration, risks/ benefits ratio) for an individual patient (in relation to age, comorbidities, expectations, lifestyle, pregnancy planning, etc.). 
- Physicians are asked to be more and more expert in the management of MS, to properly evaluate the efficacy/lack of clinical response of medications, and adequately manage adverse events (AEs), some of them rare and severe.

- Physicians, patients, patient associations, and stakeholders are solicited to design and develop new models of care to properly manage the complexity of MS.

The establishment of MS centers seems the right answer for appropriate diagnosis, comprehensive assessment, adequate monitoring, accurate selection of treatment, and meticulous follow-up of MS patients. A coordinated multidisciplinary team, including neurologist experts in MS, MS nurses, physiotherapists, psychologists, and possibly other specialists involved in MS management, is proposed as the best model to address the needs of persons with MS [2, 3]. The availability of psychological support and the delivery of information to patients have a crucial role to increase patient satisfaction, allowing them to share the most important decisions with the physician [4].

The decision-making process to choose the right medication for an individual patient is a complex task requiring careful evaluation of the results of clinical trials, up-to-date information from post-marketing data, and the capability to critically translate data from studies (that include carefully selected patients) to patients in everyday clinical practice, who are not carefully selected. The lack of head-to-head trials (with few exceptions) makes it difficult to compare the effectiveness of medications: postmarketing studies have many methodological limitations due to the unblinded evaluation of patients, the arbitrary assignment of treatment, and the possible imbalance between the populations under study; nevertheless, they can help physicians in the decision process. Systematic reviews and experts' papers also offer a valuable source of learning and facilitate the clinical update, critically summarizing the results produced by many studies.

At present the scenario of MS management shows a wide spectrum of therapeutic approaches among neurologists: a survey on treatment practices has been completed with 200
European neurologists from 11 European countries who responded to a questionnaire asking about the treatment of radiologically isolated syndrome (RIS) and clinically isolated syndrome (CIS), relapsing-remitting multiple sclerosis (RR-MS), and breakthrough disease [5]. Although a general agreement was observed for some aspects of MS treatment, namely for the treatment of RIS and CIS, responses to other questions varied considerably, highlighting the need to share some general rules for guidance of MS treatment: the benefit could be for physicians and patients, the former because of the possibility to get information from literature data critically revised by experts, the latter because of the possibility to be reassured they are treated according to the best clinical evidence, independently from confounding subjective variables. Of course, guidelines are not compulsory rules, rather useful suggestions that should be implemented in individual cases according to their clinical history, clinical and laboratory status, comorbidities, and personality.

In addition to intrinsic limitations related to the different views of physicians and patients, other limitations to appropriate access to DMDs are represented by the different delivery of care between countries, and the different positions (and frequently restrictions) of national health systems [6].

The implementation of specialty care programs for chronic disorders and the systematic application of guidelines offer a clear advantage in improving the safety and effectiveness of high-complexity, high-cost care for specialty populations, improving the quality of services and the clinical outcome [7].

The European Committee for Treatment and Research in Multiple Sclerosis (ECTRIMS) and European Academy of Neurology (EAN) have recently delivered guidelines for MS treatment [8], followed, some months later, by the guidelines of the American Academy of Neurology (AAN) $[9,10]$. Two important and highly qualified documents are thereafter now in the hands of neurologists.

Both guidelines have been realized starting from identification of the most relevant questions of MS treatment. These questions are 
reported elsewhere [11], with an overlap for some of them, but a different approach for others. To answer these questions, both guidelines have meticulously screened and critically and accurately reviewed literature data from systematic reviews (SRs) and from randomized clinical trials (RCTs), finally providing a grading of quality of evidence and of the level of recommendation.

The grading of quality of evidence for therapeutic questions was addressed by both guidelines by means of the GRADE approach (that takes into account many methodological variables such as study design, risk of bias, inconsistency, indirectness, and imprecision).

The strength of recommendations was defined by the ECTRIMS/EAN guidelines by means of a ranking scale on the basis of the quality of evidence and benefits/harms balance, and after an agreement of $80 \%$ was reached. Level of evidence and obligation was assessed by the AAN guidelines using a Delphi process and assigning the following scores: level A (strongest level of recommendation, corresponding to the verb "must"), B (lower level of strength, corresponding to the verb "should"), C (low level of evidence, corresponding to the verb "may").

The results of clinical trials were summarized by the ECTRIMS/EAN guidelines for each review question, leading to the recommendations reported in Ref. [8], for a total of 21 statements covering these topics: efficacy of DMDs, monitoring treatment response, treatment strategy if inadequate treatment response, treatment strategies in case of safety issues, long-term treatment, treatment in special situations such as pregnancy (respectively 9, 4, 2, 2, 1, 3 recommendations).

The AAN guidelines have reported separately the results of clinical trials for each medication, in relation to seven review questions [9], and the statements (or recommendations) for clinical practice in sections [10] starting diseasemodifying therapy (DMT), switching DMT, and stopping DMT (respectively 17, 10, 3 statements).

At the end of this complex process (both guidelines provide the results of their overview in the manuscript and in supplementary sections), the two groups of experts have summarized the indications for treatment and management in "Recommendations" or "Statements" $[8,10]$. An attempt has been made to identify sections with similar or different findings, which are discussed here.

As expected, the level of agreement between the two guidelines covers many areas of MS treatment, in particular regarding the treatment of subjects with CIS, RR-MS, primary progressive MS (PP-MS), and MS monitoring. To summarize:

(a) CIS It is suggested to consider the treatment with DMDs both for patients fulfilling or not fulfilling the current diagnostic criteria for MS: the latter-if showing an abnormal MRI-with IFNB or glatiramer acetate (GA), according to ECTRIMS/EAN guidelines, or with DMDs for patients with two or more brain lesions with characteristics consistent with MS, according to AAN guidelines.

(b) RR-MS Patients should be treated with any of the DMDs available, taking into consideration patient characteristics, level of disease activity, drug safety profile, accessibility of the drug (ECTRIMS/EAN guidelines), and specifically suggesting the use of alemtuzumab, fingolimod, or natalizumab for active patients (AAN guidelines). The treatment should be continued in patients who do not present clinical and MRI activity or safety concerns. AAN guidelines recommend to perform a follow-up and periodic re-evaluation of the decision to discontinue a DMD for patients who decide to stop a medication; for those evolving to secondary progressive MS (SPMS), the clinician should assess the risk of reactivation of MS and should advise discontinuation of a DMD in patients without clinical/MRI activity and high disability. According to AAN guidelines the discontinuation of a DMD should also be considered for subjects with CIS who have not been diagnosed with MS.

(c) Primary progressive MS (PP-MS) Both guidelines give the indication to consider ocrelizumab for patients with this clinical phenotype.

(d) MS monitoring Close monitoring of patients is highlighted in both guidelines, for the 
evaluation of safety and tolerability, scheduled according to the label of each medication, but also for assessment of clinical effectiveness: in addition to clinical data, treatment decisions should also consider MRI measurements. The appropriate use of MRI, in particular with reference to its standardization, time, and intervals of testing (in relation to the speed of action of each medication and safety risk factors), is discussed by ECTRIMS/EAN guidelines, whereas the AAN guidelines mainly address the criteria of no response.

(e) Switching Both guidelines consider MRI and clinical measurements to evaluate disease evolution. When and how to switch from one medication to another one? The ECTRIMS/EAN guidelines suggest to move to more efficacious drugs for patients treated with IFNB or GA who continue to present disease activity; when patients treated with a highly efficacious drug stop the treatment because of inefficacy or safety concerns, it is recommended to switch to another highly efficacious drug, taking into account the level of disease activity, pharmacokinetic profile of the previous drug, and the risk of reactivation/rebound of MS. AAN guidelines advise a switch of treatment irrespective of the ongoing drug, delineate the criteria of no response, and also consider other reasons for switching such as lack of adherence, occurrence of AEs (including infections, risk of progressive multifocal leukoencephalopathy (PML), occurrence of malignancies, tolerability issues), and development of neutralizing antibodies.

As is well known, the treatment of SP-MS is disappointing, as very poor evidence exists that DMDs are effective in patients with this clinical phenotype. The ECTRIMS/EAN guidelines address this issue suggesting a possible use of IFNB, after discussion with patients about realistic expectations in terms of effectiveness and tolerability, and use of mitoxantrone for subjects with active SP-MS, specifically discussing the safety and tolerability profile with patients. The possible use of ocrelizumab or cladribine is also suggested for active SP-MS, with a weak indication, opening the issue whether the new classification of clinical courses can lead to a reinterpretation of results of clinical trials. In contrast, AAN guidelines $[9,10]$ do not specifically address the topic of SP-MS treatment, and do not recommend the use of mitoxantrone "to people with MS unless the potential therapeutic benefits greatly outweigh the risks", and address the question whether to discontinue a DMT in people who do not have ongoing relapses or MRI activity and an Expanded Disability Status Scale (EDSS) $\geq 7$.

Some aspects of MS treatment are approached differently by the two guidelines:

- The issue of adherence is specifically addressed by the AAN but not by the ECTRIMS/EAN guidelines, as are the indication for dialogue between doctors and people with MS, to face realistic expectations of treatment, patient preferences, evaluation of risks and benefits.

- The indication for the establishment of MS centers is particularly addressed by the ECTRIMS/EAN guidelines, for appropriate and accurate assessment, monitoring of patients, and for detection and management of AEs.

- As already mentioned, the issues of switching are more widely treated by AAN guidelines, as are some specific questions about the use of natalizumab, and issues related to occurrence of serious AEs (infections, neoplasms etc.).

- As already discussed, the use of MRI is addressed in more detail by ECTRIMS/EAN guidelines.

Another topic that is addressed differently by the two guidelines is pregnancy: both guidelines advise against the use of DMDs during pregnancy; however, the ECTRIMS/EAN guidelines do not avoid the use of GA, consider continuing IFNB and GA until pregnancy is confirmed for women who are planning a pregnancy, and also consider continuing the treatment during pregnancy if at risk of MS reactivation. Finally the ECTRIMS/EAN guidelines address the question of women with highly active evolution who plan pregnancy or have an unplanned pregnancy: natalizumab is considered an option, after full discussion of possible 
risks; alemtuzumab too is given as an additional option for women with active MS who plan pregnancy, but recommending an interval of 4 months from the last infusion.

Only the AAN guidelines suggest considering the possible risks of teriflunomide and cyclophosphamide for men in relation to their reproductive plans.

Two topics that have been recently addressed by many scientific contributions are not included in either guideline: treatment with hematopoietic stem cell transplantation (HSCT) and treatment of pediatric MS. In the case of HSCT, this treatment is not officially approved despite being recognized as an effective and relatively safe treatment for MS patients with aggressive disease evolution [12]. In the case of pediatric MS, unapproved medications are currently available and the treatment is mainly supported by open-label observational studies: ECTRIMS/EAN guidelines refer to ad hoc recommendations [13].

To summarize, the two guidelines offer a similar view and similar recommendations for the most relevant and common questions of clinical practice.

For some aspects of MS treatment, as previously discussed, the positions are a bit different, in particular in relation to the use of mitoxantrone and the treatment of SP-MS, but indeed these are topics still not supported by exhaustive data. Differences are more consistent for some other questions, such as those related to pregnancy management.

As discussed by two authors who contributed to elaborate the ECTRIMS/EAN and AAN guidelines [11], these discrepancies could be explained by:

- Differences in research questions that have been differently delineated, elaborated, and adopted

- Differences in the assessment of the level of evidence

- Differences in the process of quality appraisal

Guidelines are indications that must be interpreted and translated into clinical practice, helping physicians in their clinical decision. They are the end of a process, but also the starting point for their implementation and use in clinical practice. Moreover they should be periodically updated, in consideration of new literature data and information from practical clinical use. A future objective could be to better identify and to try to unify clinical questions, starting from the current guidelines, and providing a list of common questions: in this way we could expect to have more homogeneous answers. In addition it could be meaningful to identify topics that should be better developed: for example, the definition of clinical activity, and of no response, that are not extensively discussed by current guidelines.

As a final consideration, guidelines will reach their most important goal if they are able to improve the care of patients, transcending differences among physicians and countries: each country, by means of each scientific society, should be asked to answer about their real applicability.

\section{ACKNOWLEDGEMENTS}

Funding. No funding or sponsorship was received for this study or publication of this article.

Authorship. All named authors meet the International Committee of Medical Journal Editors (ICMJE) criteria for authorship for this article, take responsibility for the integrity of the work as a whole, and have given their approval for this version to be published.

Disclosures. Angelo Ghezzi received honoraria for speaking and consultancy by Novartis, Genzyme, Roche, Merck-Serono, Teva, Mylan.

Compliance with Ethics Guidelines. This article is based on previously conducted studies and does not contain any studies with human participants or animals performed by any of the authors.

Open Access. This article is distributed under the terms of the Creative Commons Attribution-NonCommercial 4.0 International License (http://creativecommons.org/licenses/ 
by-nc/4.0/), which permits any noncommercial use, distribution, and reproduction in any medium, provided you give appropriate credit to the original author(s) and the source, provide a link to the Creative Commons license, and indicate if changes were made.

\section{REFERENCES}

1. Sorensen PS. New management algorithms in multiple sclerosis. Curr Opin Neurol. 2014;27:246-59.

2. Feys P, Giovannoni G, Dijsselbloem N, et al. The importance of a multi-disciplinary perspective and patient activation programmes in MS management. Mult Scler. 2016;22(2 Suppl):34-46.

3. NICE: Multiple sclerosis in adults: management clinical guideline. 2014. http://nice.org.uk/ guidance/cg186. Accessed May 2018.

4. Mattarozzi K, Baldin E, Zenesini C, et al. Effect of organizational features on patient satisfaction with care in Italian multiple sclerosis centres. Eur J Neurol. 2017;24:631-7.

5. Fernandez O, Delvecchio M, Edan G, et al. Survey of diagnostic and treatment practices for multiple sclerosis on Europe. Eur J Neurol. 2017;24:516-22.

6. Berger T, Adamczyk-Sowa M, Csépány $\mathrm{T}$, et al. Management of multiple sclerosis patients in central European countries: current needs and potential solutions. Ther Adv Neurol Disord. 2018;11:1-12.
7. Botts SR, Gee MT, Chang CC, et al. Design and implementation of population-based specialty care programs. Am J Health Syst Pharm. 2017;74(18):1437-45.

8. Montalban X, Gold R, Thompson AJ, et al. ECTRIMS/EAN guideline on the pharmacological treatment of people with multiple sclerosis. Mult Scler. 2018;24(2):96-120.

9. Rae-Grant A, Day GS, Ann Marrie R, et al. Comprehensive systematic review summary: diseasemodifying therapies for adults with multiple sclerosis: report of the guideline development, dissemination, and implementation subcommittee of the American Academy of Neurology. Neurology. 2018;90:789-800.

10. Rae-Grant A, Day GS, Ann MR, et al. Practice guideline recommendations summary: diseasemodifying therapies for adults with multiple sclerosis-report of the guideline development, dissemination, and implementation subcommittee of the American Academy of Neurology. Neurology. 2018;90:777-88.

11. Marrie RA, Montalban X. Disease-modifying therapy in multiple sclerosis: two guidelines (almost) passing in the night. Mult Scler J. 2018;24:558-62.

12. Mancardi G, Sormani MP, Muraro PA, et al. Intense immunosuppression followed by autologous haematopoietic stem cell transplantation as a therapeutic strategy in aggressive forms of multiple sclerosis. Mult Scler. 2018;24:245-55.

13. Chitnis T, Tenembaum S, Banwell B, et al. Consensus statement: evaluation of new and existing therapeutics for pediatric multiple sclerosis. Mult Scler. 2012;18:116-27. 